\title{
Anion co-doped Titania for Solar Photocatalytic Degradation of Dyes
}

\author{
Young-Seak Lee ${ }^{1, \uparrow}$, Sang Jin Kim ${ }^{1}$, P.Venkateswaran ${ }^{1}$, Jeen-Seok Jang ${ }^{2}$, \\ Hyuk $\mathrm{Kim}^{3}$ and Jong-Gyu Kim ${ }^{3}$
}

\author{
${ }^{1}$ Department of Fine Chemical Engineering \& Applied Chemistry Chungnam National University, Daejeon 305 764, Republic of Korea \\ ${ }^{2}$ Chung Buk Regional Small \& Med. Business Administration, Cheong Ju 418-6, Korea \\ ${ }^{3}$ Hanil Green Tech Co., LTD, Chungnam, Republic of Korea \\ •e-mail address: youngslee@cnu.ac.kr \\ (Received May 6, 2008; Accepted June 9, 2008)
}

\begin{abstract}
In order to investigate the effect of doping $\mathrm{C}, \mathrm{N}, \mathrm{B}$ and $\mathrm{F}$ elements on $\mathrm{TiO}_{2}$ for reducing the band gap, the heat treatment of $\mathrm{TiO}_{2}$ was carried out with tetraethylammonium tetrafluoroborate. Through XRD and XPS analysis, the C, N, B and F doped anatase $\mathrm{TiO}_{2}$ was confirmed. According to the increase of temperature during treatment, the particle size was increased due to aggregation of $\mathrm{TiO}_{2}$ with elements $(\mathrm{B}, \mathrm{C}, \mathrm{N}$ and $\mathrm{F}$ ). To investigate the capacity of photocatalyst for degradation of dye under solar light, the degradation of acridine orange and methylene blue was conducted. The degradation of dyes was carried out successfully under solar light indicating the effect of doping elements $(\mathrm{B}, \mathrm{C}, \mathrm{N}$ and $\mathrm{F})$ on $\mathrm{TiO}_{2}$ for reducing the band gap effectively.
\end{abstract}

Keywords : Titanium dioxide, Photocatalyst, Doping, Degradation, Band gap

\section{Introduction}

The efficient utilization of solar energy is one of the major goals of modern science and engineering that will have a great impact on technological applications [1]. As one of the materials being developed for photocatalytic applications, titanium dioxide $\left(\mathrm{TiO}_{2}\right)$ remains the most promising because of its high efficiency, low cost, chemical inertness, and photostability [2]. $\mathrm{TiO}_{2}$ represents an effective photocatalyst for water and air purification, and for self-cleaning surfaces. Additionally, it can be used as antibacterial agent due to strong oxidation activity and superhydrophilicity [3]. However, the widespread technological use of $\mathrm{TiO}_{2}$ is impaired by its wide band gap $(3.2 \mathrm{eV})$, which requires ultraviolet irradiation for photocatalytic activation. Because UV light accounts for only a small fraction (5\%) of the sun's energy compared to visible light (45\%), any shift in the optical response of $\mathrm{TiO}_{2}$ from the UV to the visible spectral range will have a profound positive effect on the photocatalytic efficiency of the material.

An initial approach for shifting the optical response of $\mathrm{TiO}_{2}$ from the UV to the visible spectral range was the doping of $\mathrm{TiO}_{2}$ with transition-metal elements [4]. However, metal doping has several drawbacks. The doped materials have been shown to suffer from thermal instability, and the metal centers act as electron traps, which reduces the photocatalytic efficiency. Furthermore, the preparation of transition-metal-doped $\mathrm{TiO}_{2}$ requires more expensive ion- implantation facilities [5]. Later, it was shown that the desired band gap narrowing of $\mathrm{TiO}_{2}$ can be better achieved by using anionic dopant species rather than metals ions. There are numerous recent reports on nonmetal-doped $\mathrm{TiO}_{2}$ systems, for example, carbon [6], nitrogen [7], boron [8] and fluorine [9] doped photocatalysts. These anions doped catalyst may be more appropriate for the extension of photocatalytic activity of $\mathrm{TiO}_{2}$ into the visible region than other methods because their impurity states are near the valance band edge, but they do not act as charge carriers, and their role as recombination centers might be minimized compared to metal cation doping [10].

Apart from doping $\mathrm{TiO}_{2}$ with a single anion, it is highly anticipated that doping $\mathrm{TiO}_{2}$ with an appropriate combination of anions would, of course, result in more visible light sensitive photocatalysts for a desired application. In this context, $\mathrm{Li}$ et al. [11] synthesized N-F-codoped $\mathrm{TiO}_{2}$ photocatalysts by spray pyrolysis using $\mathrm{TiCl}_{3}$ and $\mathrm{NH}_{4} \mathrm{~F}$ precursors and observed an enhanced photoreactivity of the materials in visible light. Recent studies indicate that C-N doped $\mathrm{TiO}_{2}$ materials exhibited the highest photocatalytic activity, which could be assigned to the synergistic effect of doped $\mathrm{C}$ and $\mathrm{N}$ atoms [12]. Codoping strategies have revealed that of $\mathrm{TiO}_{2}$ can result in the development of visible active photocatalysts [13-15].

To the best of our knowledge, there has been no publication on the codoping of carbon with nitrogen, boron and fluorine and its photocatalytic performance with solar 
Table 1. Details of the Prepared Catalyst Samples and XPS Results

\begin{tabular}{|c|c|c|c|c|c|c|c|c|c|}
\hline \multirow{2}{*}{$\begin{array}{l}\text { S. } \\
\text { No. }\end{array}$} & \multirow{2}{*}{$\begin{array}{l}\text { Sample } \\
\text { code }\end{array}$} & \multirow{2}{*}{$\begin{array}{c}\text { TEATFB } \\
\text { concentration }(\mathrm{M})\end{array}$} & \multirow{2}{*}{$\begin{array}{c}\text { Calcination } \\
\text { temperature }(\mathrm{C})\end{array}$} & \multicolumn{6}{|c|}{ Elemental composition (\%) } \\
\hline & & & & $\mathrm{Ti}$ & $\mathrm{O}$ & $\mathrm{C}$ & $\mathrm{N}$ & $\mathrm{B}$ & $\mathrm{F}$ \\
\hline 1 & Undoped $\mathrm{TiO}_{2}$ & - & - & 30.6 & 69.4 & 0 & 0 & 0 & 0 \\
\hline 2 & S1-A & 0.1 & 400 & 19.6 & 56.6 & 16.1 & 0.5 & 5.8 & 1.4 \\
\hline 3 & S1-B & 0.1 & 600 & 20.1 & 56.6 & 16.6 & 0.3 & 5.1 & 1.3 \\
\hline 4 & S1-C & 0.1 & 800 & 19.4 & 57.3 & 16.3 & 0.2 & 5.7 & 1.1 \\
\hline 5 & S1-D & 0.1 & 1000 & 16.9 & 56.3 & 20.5 & 0 & 6.2 & 0.1 \\
\hline
\end{tabular}

light. In this letter, we report $\mathrm{C}, \mathrm{N}, \mathrm{B}$ and $\mathrm{F}$ co-doped $\mathrm{TiO}_{2}$ photocatalyst preparation, its characterization and the photocatalytic properties of these new photocatalysts. The photoreactivity of the codoped $\mathrm{TiO}_{2}$ was evaluated for the degradation of textile dyes with solar light irradiation.

\section{Experimental}

\subsection{Materials}

Anatase titanium dioxide ( $99 \%$ Acros) of average particles size $200 \mathrm{~nm}$, tetraethylammonium tetrafluoroborate $\left(\left(\mathrm{CH}_{3} \mathrm{CH}_{2}\right)_{4} \mathrm{~N}^{+} \mathrm{BF}_{4}^{-}\right.$, $99 \%$, Aldrich) were used as received without further purification. Dyes namely methylene blue (CI number- 52015), and acridine orange (CI number- 46005) were purchased from Acros (USA). Deionised water was used for preparing the reagents.

\subsection{The preparation of samples}

Carbon, nitrogen, boron and fluorine co-doped $\mathrm{TiO}_{2}$ powder was obtained by mixing $10 \mathrm{~g}$ of $\mathrm{TiO}_{2}$ with $25 \mathrm{ml}$ of $0.1 \mathrm{M}$ tetraethylammonium tetrafluoroborate (TEATFB) precursor. The mixture was stirred at room temperature for overnight and allowed to settle. The obtained powders were separated by filtration and dried at $110^{\circ} \mathrm{C}$. Then, the resulting powder was calcined at temperature in the range of 400 $1000^{\circ} \mathrm{C}$ for $2 \mathrm{~h}$ in absence of air. The calcination temperature was attained at a heating rate of about $10^{\circ} \mathrm{C} / \mathrm{min}$. The resulting powder was washed three times with distilled water to remove undoped salt and finally dried under air at $110^{\circ} \mathrm{C}$. Pure $\mathrm{TiO}_{2}$ was used as the reference sample. The details of sample code, dopant concentration and calcination temperature of the prepared catalysts are presented in Table 1.

\subsection{Characterization}

A Scanning Electron Microscope (SEM, JSM-6300, JEOL Ltd, Japan) was used to explore the surface properties of the samples under $20 \mathrm{keV}$. The SEM analysis was conducted by a gold- palladium sputtering process. The crystalline phases of samples were determined by X-ray diffraction (XRD, D/ MAX-2200 Ultima/PC, RigaKu, USA) with $\mathrm{Cu} \mathrm{K \alpha}$ radia- tion The X-ray Photoelectron Spectroscopy (XPS, MultiLab 2000 spectrometer, Thermo electron corporation, England) spectra were obtained with $\mathrm{Al} \mathrm{K \alpha}$ excitation to know the changes of chemical species on the samples' surface before and after doping process. IR spectra were recorded on a FTIR instrument (Shimadzu (IR Prestige- 21), Japan) pelletizing the samples with $\mathrm{KBr}$.

To evaluate photocatalytic activity of the prepared catalyst, $50 \mathrm{ml}$ of aqueous solution containing dye was placed in a glass beaker with $100 \mathrm{mg}$ of photocatalyst. The suspension was irradiated in solar light. The solar intensity was measured with a digital illumination meter (INS, DX-200). Few millilitre aliquot of the aqueous suspension were collected at regular time periods during irradiation and filtered through syringe filters to remove catalyst particles. Dye concentration was estimated by spectrophotometric method at 660 and $493 \mathrm{~nm}$ for methylene blue and acridine orange respectively.

\section{Results and discussion}

\subsection{The surface morphology of sample}

Catalysts obtained by calcinations at 400 to $800^{\circ} \mathrm{C}$ were in pale yellowish color, intensity decreases with increase in temperature and sample calcined at $1000^{\circ} \mathrm{C}$ was bluish in color. This blue color may be due to reduction of $\mathrm{Ti}^{4+}$ to $\mathrm{Ti}^{3+}$. The study of Grey et al. suggested that boron incorporation into rutile $\mathrm{TiO}_{2}$ led to partial reduction of $\mathrm{Ti}^{4+}$ to $\mathrm{Ti}^{3+}$ [4]. Figure 1 represents the typical SEM images of undoped $\mathrm{TiO}_{2}$ and $\mathrm{C}, \mathrm{N}, \mathrm{B}$ and $\mathrm{F}$ elements doped sample calcined at 800 and $1000^{\circ} \mathrm{C}$ respectively. It can be observed that the aggregation of particles in the doped samples than the undoped $\mathrm{TiO}_{2}$. The lattice parameters changes little after doping in $\mathrm{TiO}_{2}$ particles. It is inferred that $\mathrm{C}$ atoms are difficult to weave into the $\mathrm{TiO}_{2}$ lattice, and most likely exist on the surface of $\mathrm{TiO}_{2}$ particles and form a layer composed of complex carbonate species, resulting in the suppression of crystal growth. Chen et al. proposed that doped carbon species from tetrabutylammonium as carbon precursor, $\mathrm{C}$ can locate at the surface of $\mathrm{TiO}_{2}$ particles as $\mathrm{C}_{3}-\mathrm{TiO}_{2}$ and acts as a role of linker between aggregated particles [8].

A clear relationship between the doped $\mathrm{TiO}_{2}$ and calcina- 

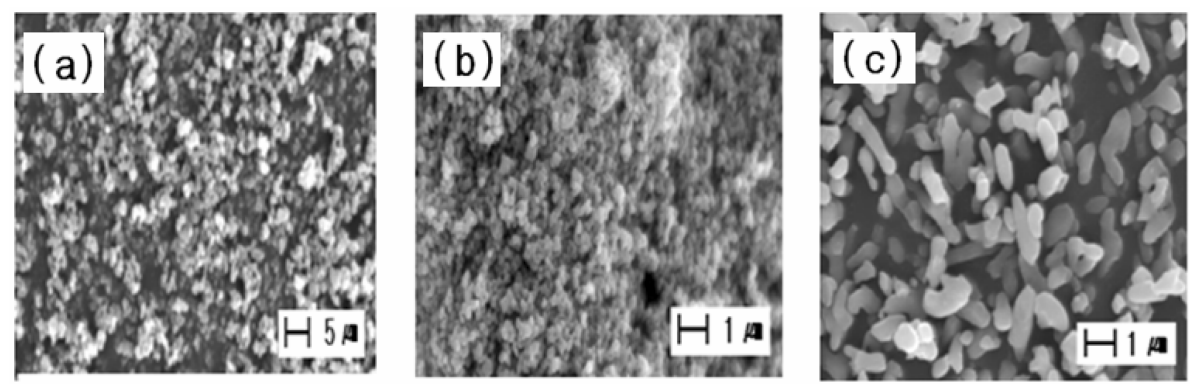

Fig. 1. SEM images of samples (a) Undoped $\mathrm{TiO}_{2}$, (b) S1-C, (c) S1-D.

tion temperature is observed at $1000^{\circ} \mathrm{C}$. As the calcination temperature increases, the microcrystal size of $\mathrm{TiO}_{2}$ increases because of the fusion of $\mathrm{TiO}_{2}$ particles, in good agreement with the XRD results.

\subsection{XRD patterns of samples}

In order to study the effect of concentration of the precursor material, attempts have been made with $0.1,0.25$ and $0.5 \mathrm{M}$ TEATFB doped $\mathrm{TiO}_{2}$ catalysts. Preliminary studies on degradation of dyes reveal that, 0.1-0.5 M TEATFB doped $\mathrm{TiO}_{2}$ catalysts showed uniform degradation efficiency. Hence, $0.1 \mathrm{M}$ TEATFB doped catalyst alone investigated for their characterization and applications. The UV-diffuse reflectance spectra (not shown here) of doped samples indicated a strong absorption in the visible range (400-600 nm) when compared to pristine $\mathrm{TiO}_{2}$. The XRD patterns revealed that the samples heat treated up to $800^{\circ} \mathrm{C}$ were present in photoactive anatase form in Fig. 2.

\subsection{XPS analysis}

The XPS binding energies of the core level in all the samples indicate the successful incorporation of the dopants

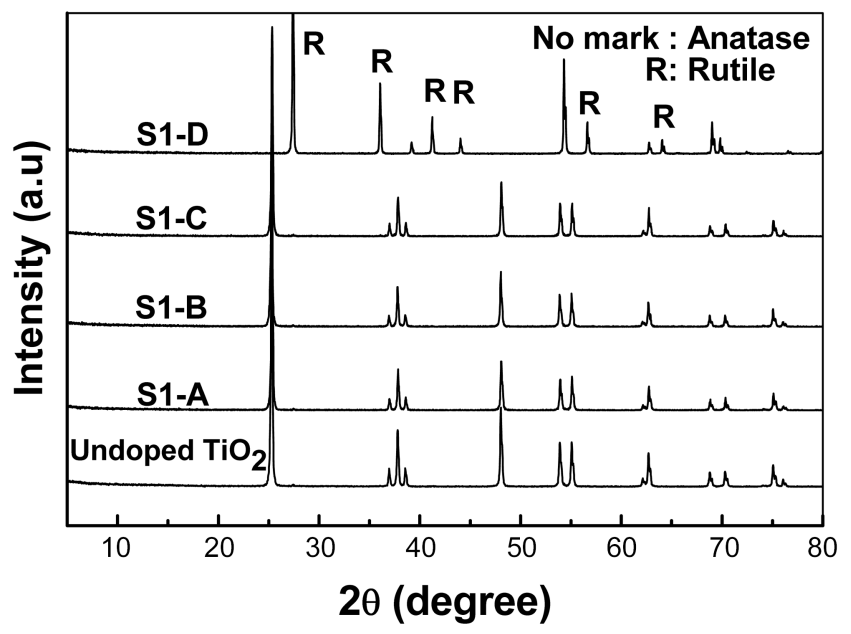

Fig. 2. XRD patterns of samples. into the lattice. The atomic composition of pure and doped $\mathrm{TiO}_{2}$ samples is presented in Table 1. The results show that all the doped samples have C, N, B and F elements, where as it is absent in undoped $\mathrm{TiO}_{2}$.

The XPS spectra of carbon in doped titania samples is shown in Fig. 3. In all the samples peaks around $285 \mathrm{eV}$ is attributed to $\mathrm{C} 1 \mathrm{~s}$ of adventitious elemental carbon [16]. No appreciable signal related to Ti-C bond $(281.5 \mathrm{eV})$ was observed, indicating the amount of this bond was very low in these samples.

The active site and nitrogen state responsible for the enhanced photocatalytic activity of nitrogen-doped $\mathrm{TiO}_{2}$. In the present study, the $\mathrm{N} 1 \mathrm{~s}$ peak at $400 \mathrm{eV}$ was observed in the doped $\mathrm{TiO}_{2}$ samples. Similarly, a significant peak at around $402 \mathrm{eV}$ and a minor peak at around $400 \mathrm{eV}$ assigned to $\mathrm{N} 1 \mathrm{~s}$ peak were reported in $\mathrm{N}$-doped $\mathrm{TiO}_{2}$ [17]. Sakthivel and Kisch [18] prepared the nitrogen doped titania from titanium tetraisopropoxide or titanium tetrachloride and thiourea. They observed the binding energy of $400.1 \mathrm{eV}$ assigned to hyponitrite at the surface, which attributed to the doped nitrogen into the $\mathrm{TiO}_{2}$. The sample (S1-A) calcined at $400^{\circ} \mathrm{C}$ exhibits $\mathrm{N} 1 \mathrm{~s}$ signal and no peak is observed in the sample S1-D. The fact that nitrogen was observable in the three samples, the atomic percentage of $\mathrm{N}$ decreases with increase in calcination temperature. This suggests that this

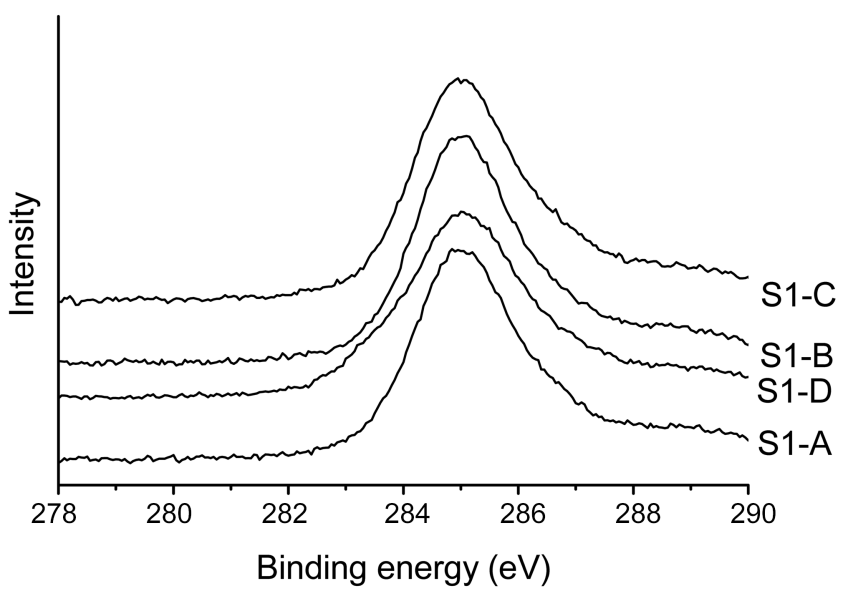

Fig. 3. XPS spectra of $\mathrm{C} 1 \mathrm{~s}$ for the doped $\mathrm{TiO}_{2}$ samples. 


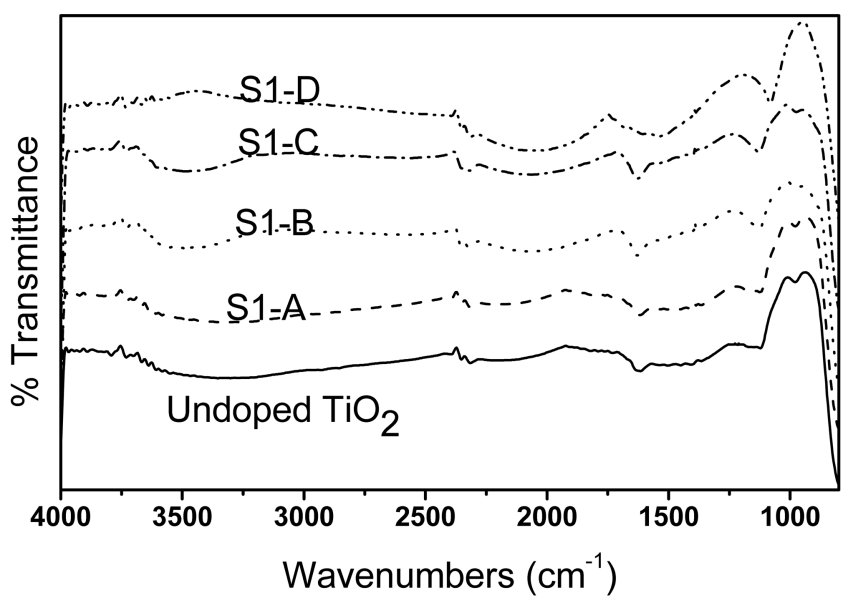

Fig. 4. FT-IR spectra of $\mathrm{TiO}_{2}$ particles before and after doping at various temperatures.

species is formed in the initial calcination temperatures and subsequently lost. In accordance with this result, the higher temperature calcinations afford to loss of nitrogen. The doping of $\mathrm{C}$ is more in all the samples followed by $\mathrm{B}, \mathrm{F}$ and $\mathrm{N}$.

It is observed that the B 1s region contains one peak only, and the binding energy shifts from $191.1 \mathrm{eV}$. This implies the formation of weak bond in Ti-B, also due to the interference effect of other elements bonded with Ti. Taking into account the standard binding energy of $\mathrm{B} 1 \mathrm{~s}$ in $\mathrm{TiB}_{2}$ (187.5 eV, B-Ti bond), this result displays that the boron atom is probably incorporated with $\mathrm{TiO}_{2}$ to some extent, and the chemical environment surrounding boron is similar to that of $\mathrm{TiB}_{2}[18]$.

A main peak of the asymmetrical line shape in F1s spectrum observed can be assigned to structures containing oxyfluoride (F-Ti-O) functional groups while the shoulder at $685 \mathrm{eV}$ is connected to F-Ti bond [19]. These findings reveal that fluorine is incorporated in the $\mathrm{TiO}_{2}$ by substitution of oxygen atoms in its lattice. The fluorine ions are incorporated in the $\mathrm{TiO}_{2}$ lattice will attribute to the improved $\mathrm{TiO}_{2}$ crystallinity [20], as well as inhibit the phase transformation from anatase to rutile. This is because the ligand prevents the condensation of spiral chains of anatase $\mathrm{TiO}_{6}$ octahedra to form linear chains of rutile $\mathrm{TiO}_{6}$ octahedra, while growth of the anatase spiral chains is still possible [21]. In the case of $\mathrm{C}$ and $\mathrm{F}$ there is no observable peak shift. This is due to the strong bonding of $\mathrm{F}, \mathrm{C}$ atoms with Ti particles.

\subsection{FT-IR analysis}

The FT-IR spectra of undoped and doped $\mathrm{TiO}_{2}$ samples in the frequency range of $1000-4000 \mathrm{~cm}^{-1}$ were recorded (Fig. 4). A peak around $1200 \mathrm{~cm}^{-1}$ can be observed, which belongs to the $\mathrm{B}-\mathrm{O}$ bond in the $\mathrm{B}_{2} \mathrm{O}_{3}$ crystal. Another peak around $1400 \mathrm{~cm}^{-1}$ is present in the IR spectra, attributed to the Ti-O-B bond [24] in the prepared sample. In the low

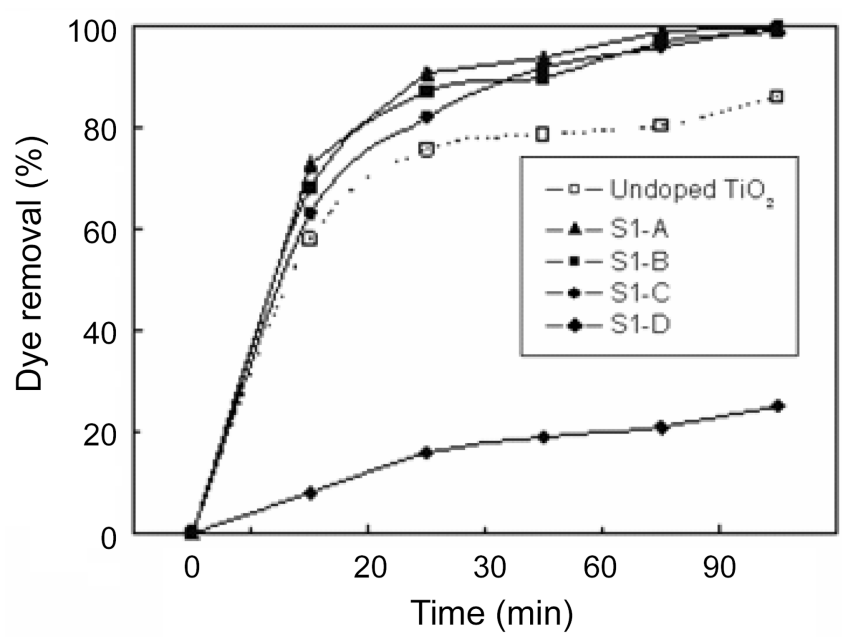

(a) Removal of acridine orange dye

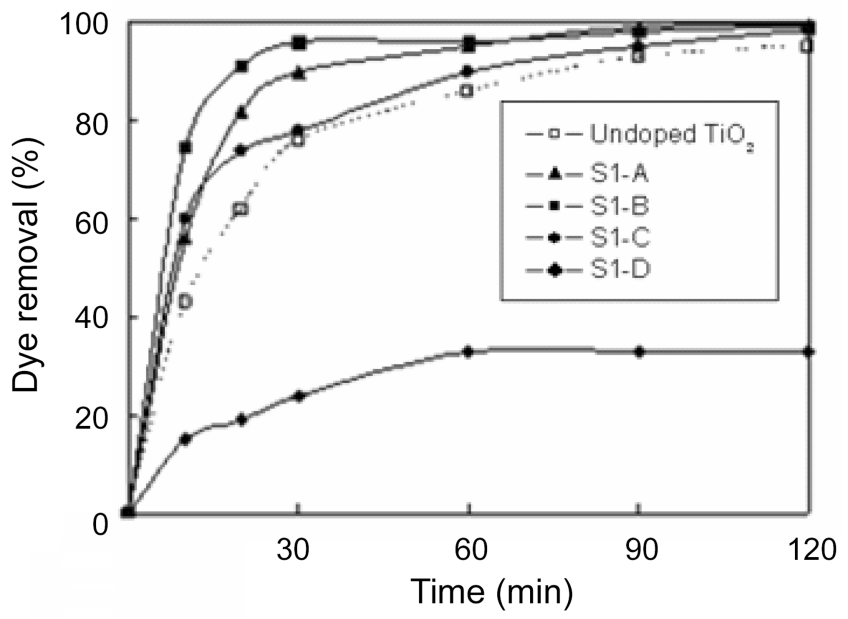

(b) Removal of methylene blue dye

Fig. 5. Photomineralization of the dye with solar light; (conditions: Volume of dye sample $=50 \mathrm{ml}, \mathrm{TiO}_{2}$ concentration $=0.1 \mathrm{~g}$, Dye concentration $=10 \mathrm{ppm})$.

frequency part of the spectra, many fluctuant bands develop around $500 \mathrm{~cm}^{-1}$ are attributed to the anatase phase of $\mathrm{TiO}_{2}$ [23]. The hyponitrite peak at $1385 \mathrm{~cm}^{-1}$ corresponds to the binding energy of $400 \mathrm{eV}$ in XPS. The peak around 1719 $\mathrm{cm}^{-1}$ attributed to the formation of carbonate corresponding to the binding energy of $287.9 \mathrm{eV}$ in XPS. Similar results were reported for the $\mathrm{C}$ and $\mathrm{N}$ doped $\mathrm{TiO}_{2}$ films [24]. The FT-IR results indicate the doping of nitrogen and carbon and IR spectrum are consistent with that of XPS. The presence of F is not clearly identified by the FT-IR analysis.

\subsection{The capacity of photo degradation}

To explore the visible-light photocatalytic activity of the doped $\mathrm{TiO}_{2}$ photocatalyst, removal of two dyes namely acridine orange and methylene blue were investigated. All 
the experiments were carried out in solar light with intensity $4 \sim 8 \times 10^{4}$ lux. Figure 5 illustrates the decolorization of the dye with solar light. In both the dyes studied, the $\mathrm{TiO}_{2}$ calcined at $400^{\circ} \mathrm{C}$ (S1-A) shows effective colour removal in short time, when comparing with samples calcined at higher temperatures. Fig. 5(a) illustrates the visible light activity of codoped $\mathrm{TiO}_{2}$ on the removal of dye acridine orange. After 1 $\mathrm{h}$ of exposure to the samples to solar light more than $95 \%$ colour was removed in samples S1-A, S1-B, S1-C and only $20 \%$ in sample $\mathrm{S} 1-\mathrm{D}$ where as, undoped and blank $\mathrm{TiO}_{2}$ (in dark) induced only 59 and $11 \%$ colour removal in acridine orange dye.

The changes in concentration of methylene blue recorded during visible light irradiation at specific time interval is shown in Fig. 5(b). Experimental results show that $84 \%$ of colour is removed in solar irradiated sample after $1 \mathrm{~h}$. It can be noted that the decrease in colour in the blank sample is due to adsorption of dye by the catalyst, which may not be degraded further or little evident from the change of colour in the catalyst. In the case of doped samples the mechanism is adsorption followed by degradation. The degradation in the doped samples is 95, 96 and $90 \%$ in S1-A, S1-B and S1$\mathrm{C}$ respectively. Photocatalysis of rutile $\mathrm{TiO}_{2}$ (S1-D) showed about $33 \%$ of colour removal. Chen et al [8] reported that the degradation of methylene blue dye $\left(1.8 \times 10^{-5} \mathrm{M}\right)$ in $7 \mathrm{~h}$ using $\mathrm{C}, \mathrm{N}$ doped $\mathrm{TiO}_{2}$ catalyst $(125 \mathrm{mg})$. Comparing this result with the obtained result, the $\mathrm{C}, \mathrm{N}, \mathrm{B}$ and $\mathrm{F}$ doped catalyst shows enhanced efficiency of 3 times faster and with $20 \%$ less quantity of catalyst. The low activity of S1D (rutile type) may be due to fast recombination of generated electrons $\left(\mathrm{e}^{-}\right)$and holes $\left(\mathrm{h}^{+}\right)$. The sample calcined at $400^{\circ} \mathrm{C}$ has the highest photocatalytic activity, since a higher temperature can increase the particle size leading to decreased activity.

Comparing the obtained experimental results with the results reported earlier for methylene blue [6], the present study involving $\mathrm{C}, \mathrm{N}, \mathrm{B}$ and $\mathrm{F}$ shows enhanced dye removal. The doping of C, N, B would narrow the band gap, by the replacement of one $\mathrm{O}$ atom with the dopant atom. Fluorine doping can generate oxygen vacancies in $\mathrm{TiO}_{2}$. It is well known that oxygen vacancies in a solid will probably make the spectral limit of a surface photochemical process redshift to visible region [25]. In addition the sample calcined at $400^{\circ} \mathrm{C}$ posses a complex mixture of active carbon and carbonate species at the surface of $\mathrm{TiO}_{2}$ [26]. The obtained results suggest that the carbonaceous species formed at the surface of doped $\mathrm{TiO}_{2}$ samples could absorb more dye molecules than the undoped $\mathrm{TiO}_{2}$, besides the capability of exciting by visible light like organic dyes. When the adsorbed dye molecules are degraded, other dye molecules adsorbed on the carbonaceous layer can transfer to the residual vacancies through surface diffusion, which may be faster process than free diffusion in solution. It is well known that, for effective degradation, the organic substance should be preconcentrated on the surface of the semiconductor particles to effectively utilize the photoexcitation [27].

\section{Conclusions}

In conclusion, we reported the codoping of anions C, N, B and $\mathrm{F}$ from a single nonmetal precursor (TEATFB) in $\mathrm{TiO}_{2}$ particles with highly visible light photoactivity. XPS and FTIR analyses of the codoped samples confirmed the presence of these elements in doped titania. Possibly, the well dispersed anions promote the electron hole separation and subsequently enhance the photoactivity. The codoped $\mathrm{TiO}_{2}$ catalysts degrade the dyes faster than the undoped catalyst under solar light illumination indicating the effect of doping C, N, B and $\mathrm{F}$ on $\mathrm{TiO}_{2}$ to reduce the band gap.

\section{References}

[1] Khan, S. U. M.; Akikusa, J. J. Phys. Chem. B 1999, 103, 7184.

[2] Schiavello, M., Dordrecht, H., "Photoelectrochemistry, Photocatalysis, and Photoreactors: Fundamentals and Developments", Kluwer Academic, Boston, MA, 1985.

[3] Bhatkhande, D. S.; Pangarkar, V. G.; Beenackers A. ACM. J.Chem Technol Biotechnol 2001, 77, 102.

[4] Chiou, C. H.; Juang, R. S. Journal of Hazardous Materials 2007, 149, 1.

[5] Yamashita, H.; Honda, M.; Harada, M.; Ichihashi, Y.; Anpo, M.; Hirao, T.; Itoh, N.; Iwamoto, N. J. Phys. Chem. B 1998, 102, 10707.

[6] Palanivelu, K.; Im, J. S.: Lee, Y. S. Carbon Science 2007, 8, 214.

[7] Qiu, X.; Burda, C. Chemical Physics 2007, 339, 1.

[8] Chen, D.; Yang, D.; Wang, Q.; Jiang, Z. Ind. Eng. Chem. Res 2006, 45, 4110.

[9] Park, H.; Choi, W. J. Phys. Chem., B 2004, 108, 4086.

[10] H.Wang, J.P.Lewis J.Phys.: Condens. Matter 2005, 17, 209.

[11] Li, D.; Haneda, H.; Hishita, S.; Ohashi, N. Chem. Mater 2005, 17, 2596.

[12] Chen, D.; Jiang, Z.; Geng, J.; Wang, Q.; Yang, D. Ind. Eng. Chem. Res 2007, 46, 2741.

[13] Gombac, V.; Rogatis, L. D.; Gasparotto, A.; Vicario, G.; Montini, T.; Barreca, D.; Balducci, G; Fornasiero, P.; Tondello, E.; Graziani, M. Chemical Physics 2007, 339, 111.

[14] Li, D.; Haneda, H.; Hishita S.; Ohashi, N. Chem. Mater. 2005, 17, 2588.

[15] Yang, J.; Bai, H.; Tan, X.; Lian, J. Applied Surface Science 2006, 253, 1988.

[16] Ren.W,Ai.Z.Jia.F,Zang.L Fan.X.Zou.Z. Appl. Cata.B Env. 2007, 18, 105604. 
[17] Chen, C.; Bai, H.; Chang, S.; Chang, C.; Den, W. J. Nanopart. Res. 2006, 365.

[18] Sakthivel, S.; Kisch, H. Chem. Phys. Chem. 2003, 4, 487

[19] Swanepoel, R.; Phys, J. J. E. J. Sci .Instrum. 1983, 16, 1214.

[20] Yamaki, T.; Shumita, T.; Yamamoto, S. J. Mater. Sci. Lett. 2002, 21, 33.

[21] Izumi, F.; Bull. Chem. Soc. Jpn. 1978, 51, 1771.

[22] Sagawa, T.; Sueyoshi, R.; Kawaguchi, M.; Kudo, M.; Ihara, H.; Ohkubo, K. Chem. Commun. 2004, 7, 814.
[23] Kumar, P. M.; Badrinarayanan, S.; Sastry. M. Thin solid films 2000, 358, 122.

[24] Yang, J.; Bai, H.; Tan, H.; Lian, J. Applied Surface Science 2006, 2531988.

[25] Volodin, A. M. Catal. Today 2000, 58, 103.

[26] Reddy, K. M.; Baruwati, B.; Jayalakshmi, M.; Rao, M. M.; Manorama. S. V. Journal of Solid State Chemistry 2005, 178, 3352.

[27] Wang, W. D.; Serp, P.; Kalck, P.; Faria, J. L. Appl. Catal. B 2005, 56, 305. 